\title{
Investigating the dynamical history of the interstellar object 'Oumuamua
}

\author{
Piotr A. Dybczyński ${ }^{1}$ and Małgorzata Królikowska ${ }^{2}$ \\ ${ }^{1}$ Astronomical Observatory Institute, Faculty of Physics, A. Mickiewicz University, Słoneczna 36, Poznań, Poland \\ e-mail: dybol@amu.edu.pl \\ ${ }^{2}$ Space Research Centre of Polish Academy of Sciences, Bartycka 18A, Warszawa, Poland \\ e-mail: mkr@cbk.waw.pl
}

Received 16 November 2017 / Accepted 26 January 2018

\begin{abstract}
Here we try to find the origin of 1I/2017 U1 'Oumuamua, the first interstellar object recorded inside the solar system. To this aim, we searched for close encounters between 'Oumuamua and all nearby stars with known kinematic data during their past motion. We had checked over 200 thousand stars and found just a handful of candidates. If we limit our investigation to within a $60 \mathrm{pc}$ sphere surrounding the Sun, then the most probable candidate for the 'Oumuamua parent stellar habitat is the star UCAC4 535-065571. However GJ 876 is also a favourable candidate. However, the origin of 'Oumuamua from a much more distant source is still an open question. Additionally, we found that the quality of the original orbit of 'Oumuamua is accurate enough for such a study and that none of the checked stars had perturbed its motion significantly. All numerical results of this research are available in the appendix.
\end{abstract}

Key words. methods: numerical - celestial mechanics - comets: general - interplanetary medium

\section{Introduction}

The problem of the lack of evident interstellar visitors in our solar system has been discussed for decades. Recently, Engelhardt et al. (2017) considered the implications of not observing such interstellar visitors. Now, the situation has changed.

The first interstellar small body penetrating our solar system was discovered on Pan-STARRS1 images taken on 2017 Oct. 18.5 UT at mag 19.8 (MPC CBET 4450). Initially, it was designated as a comet (C/2017 U1) due to its near-parabolic orbit. Later on, due to the lack of any cometary activity it was renamed as A/2017 U1 (M.P.E.C. 2017-U183, issued on 2017 Oct. 25, 22:22 UT). Ten days later, in M.P.E.C. 2017-V17, issued on Nov. 6, 21:00 UT, a new concept for naming such unusual objects was announced and accordingly, A/2017 U1 was renamed as 1I/2017 U1 ('Oumuamua).

The unique dynamical nature of this object was first noted by Bill Gray in his Oct. 25 posting to the Minor Planet Mailing list $(\text { MPML })^{1}$. He obtained a preliminary orbit based on a six-day arc and noticed an atypically high eccentricity of approximately 1.2. 'Oumuamua travels at a relatively high velocity with respect to the Sun (on the order of $25 \mathrm{~km} \mathrm{~s}^{-1}$ ). Several preprints on the kinematics of this extraordinary object have recently appeared. Mamajek (2017) analysed the stars nearest the Sun for similar spatial velocity while Gaidos et al. (2017) suggested the origin of 'Oumuamua in a nearby young stellar cluster.

'Oumuamua seems to be unique for its physical characteristics as well. Meech et al. (2017) estimated its shape to be extremely elongated while Fraser et al. (2017) and Drahus et al. (2017) determined it to be a tumbling body. An interesting paper

https://groups.yahoo.com/neo/groups/mpml/info on the determination of physical parameters for 'Oumuamua has also been presented recently by Jewitt et al. (2017).

Since the nature of this object is still unknown, it might be desirable to study its dynamical history before entering the solar system interior.

This paper is organized as follows: the following section describes the model of solar vicinity dynamics which we use to track 'Oumuamua's past motion. The main task was to collect data on potential stellar perturbers. Section 3 presents the results of our numerical experiments. In the last section, we interpret these results and discuss their importance. In appendices we present complete numerical results, all stellar parameters used in this work with their references, and several examples of the geometry of the Qumuamua - star encounters.

\section{Approach to the problem}

To analyze the interstellar path of 'Oumuamua in the solar neighbourhood it is necessary to numerically integrate its equations of motion, taking into account both the overall Galactic potential and all important individual stellar perturbations from the known nearby stars.

To work with contemporary stellar data, we searched the whole SIMBAD astronomical objects database ${ }^{2}$ for all stars with known positions, proper motions, parallaxes and radial velocities. To make sure we were working only with reliable data, we ensured that parallaxes were positive and radial velocities were $\leqq 500 \mathrm{~km} \mathrm{~s}^{-1}$ in modulus. The result of this search, performed on 2017 Nov. 5, consists of 201763 individual objects. Such a great number is the result of large observational projects, mainly Gaia (Gaia Collaboration 2016b) and RAVE (Kunder et al. 2017). As

2 http://simbad.u-strasbg.fr/simbad/ 
Table 1. Osculating heliocentric orbit of 'Oumuamua, based on 118 positional observations spanning the interval from 2017 Oct. 14 to 2017 Nov. 10 , available at MPC on 2017 Nov. 12

\begin{tabular}{lr}
\hline \hline Perihelion distance [AU] & $0.255234 \pm 0.000062$ \\
Eccentricity & $1.199236 \pm 0.000164$ \\
Inverse of the semimajor axis [AU ${ }^{-1}$ ] & $-0.780603 \pm 0.000618$ \\
Time of perihelion passage [TT] & 2017 Sept. $09.488519 \pm 0.001243$ \\
Inclination [deg] & $122.677069 \pm 0.005823$ \\
Argument of perihelion [deg] & $241.683487 \pm 0.011254$ \\
Longitude of the ascending node [deg] & $24.599729 \pm 0.000264$ \\
Epoch of osculation [TT] & 2017 Sept. 4.0 = JD 2458000.5 \\
\hline
\end{tabular}

Notes. Equator and ecliptic of $\mathrm{J} 2000$ is used. The obtained RMS is 0.35 arcsec.

Table 2. Barycentric original and future 'Oumuamua orbit elements.

\begin{tabular}{lrr}
\hline \hline \multicolumn{1}{c}{ Element } & \multicolumn{1}{c}{ Original orbit } & \multicolumn{1}{c}{ Future orbit } \\
\hline Perihelion distance [AU] & $0.252062 \pm 0.000063$ & $0.257286 \pm 0.000063$ \\
Eccentricity & $1.196488 \pm 0.000164$ & $1.200366 \pm 0.000167$ \\
Time of perihelion passage [TT] & 2017 Sept. 09.118037 \pm 0.001262 & 2017 Sept. 09.310111 \pm 0.001250 \\
Inverse of the semimajor axis [AU ${ }^{-1}$ ] & $-0.779521 \pm 0.000456$ & $-0.778771 \pm 0.000455$ \\
Inclination [deg] & $122.725937 \pm 0.005995$ & $122.870243 \pm 0.005900$ \\
Argument of perihelion [deg] & $241.696866 \pm 0.011361$ & $241.842028 \pm 0.011433$ \\
Longitude of the ascending node [deg] & $24.251515 \pm 0.000251$ & $24.747600 \pm 0.000256$ \\
Epoch of osculation [TT] & 1973 Oct. 05 & 2061 Aug 04 \\
\hline
\end{tabular}

it concerns a homogeneity of the data taken from the SIMBAD database we observe that $84 \%$ of astrometric measurements of these 201763 stars were copied from the TGAS catalogue (Gaia Collaboration 2016a) and another 11\% from the HIP2 catalogue (van Leeuwen 2007). The situation is slightly more complicated with radial velocity sources but still the majority of measurements $(70 \%)$ were taken from RAVE data releases (Kunder et al. 2017; Kordopatis \& RAVE Collaboration 2014) and another $11 \%$ from the Pulkovo compilation (Gontcharov 2006). The remaining radial velocity measurements taken by us from the SIMBAD database were copied from a large number of individual papers.

This stellar data set allowed us to perform accurate calculations, namely the numerical integration of 'Oumuamua's motion. To account for mutual stellar gravitational interactions, we had to integrate the $N$-body problem, consisting of 'Oumuamua, the Sun, and all individual stellar perturbers, all of which are under the influence of the overall Galactic potential. However, integrating the simultaneous motion's of over 200000 bodies would be a waste of time - a great majority of these bodies never came close enough to 'Oumuamua to disturb its motion. Instead we first prepared a short list of perturbers; see below.

Numerical integration of motion was performed in a righthanded, non-rotating, rectangular Galactocentric frame with the $O X$ axis directed in the opposite direction to the Sun's position at the starting time. We use the Model I variant of the Galactic gravitational potential described by Irrgang et al. (2013). As the starting position and velocity of the Sun we use vectors: $\boldsymbol{R}_{\odot}=\left(x_{\odot}, y_{\odot}, z_{\odot}\right)=(-8400,0,17)$ in pc and $\dot{\boldsymbol{R}}_{\odot}=(u, v, w)=$

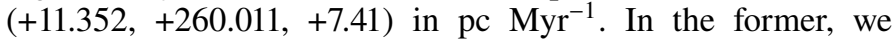
adopted the vertical position of the Sun given by Joshi (2007). A detailed description of the Galactic reference frame orientation and the Galactic potential form and parameters can be found in Dybczyński \& Berski (2015). Here we use exactly the same dynamical model and equations of motion.

The starting position and velocities of 'Oumuamua for dynamical calculations outside a planetary zone were obtained from its original orbit. We determined this orbit elements from all positional observations available in the MPC database ${ }^{3}$ on 2017 Nov. 12. Through careful data processing, we obtained an osculating orbit given in Table 1. Next, in order to observe the uncertainties in the motion of 'Oumuamua at every stage of our research, we cloned its orbit and built a swarm of 10000 orbits resembling the observations, using a method described by Sitarski (1998) which fully utilises the covariance matrix obtained during the orbit determination. Then, we numerically propagated all of these orbits forward and backward up to a heliocentric distance of $250 \mathrm{AU}$ (the distance at which planetary perturbations are negligible). The resulting barycentric elements of the original and future orbits of 'Oumuamua, along with their uncertainties, are presented in Table 2 . We used the barycentric positions and velocities of each individual clone of 'Oumuamua at 250 au as starting data for a dynamical study of this body under the gravitational influence of stars and the full Galactic potential.

We suppose that due to the lack of cometary activity, non-gravitational forces (we found them non-detectable from positional data) could not have changed the orbit of 'Oumuamua significantly during its close perihelion passage and that the original orbit is rather reliable, with the uncertainties presented in Table 2. To observe how these uncertainties influence the minimal distance between 'Oumuamua and all stars included in our model, we repeated our numerical integration for all 10000 clones of 'Oumuamua. Each encounter parameters obtained from this complex calculation as well as their variation intervals are presented in Table A.3.

However, the most important source of the close passage distance uncertainty, not estimated in this paper, is the stellar data errors. This cannot simply be modelled by the simultaneous drawing of $N$ clones for all 57 stars and 'Oumuamua because that would require $N^{58}$ numerical integrations. In this paper,

\footnotetext{
3 http://www.minorplanetcenter.net/db_search
} 
Table 3. All close encounters between 'Oumuamua and a star or stellar system closer than 1 pc obtained from the 59B model.

\begin{tabular}{lrrrrc}
\hline \hline Star name & $\begin{array}{r}\text { Min. distance } \\
{[\mathrm{pc}]}\end{array}$ & $\begin{array}{r}\text { Epoch } \\
{[\mathrm{Myr}]}\end{array}$ & $\begin{array}{r}\text { Rel. velocity } \\
{\left[\mathrm{km} \mathrm{s}^{-1}\right]}\end{array}$ & $\begin{array}{r}r_{\text {_hel }}[\mathrm{pc}] \\
\text { HIP 3757 }\end{array}$ & $\begin{array}{r}\text { Miss-distance interval } \\
{[\mathrm{pc}]}\end{array}$ \\
GJ 4274 & 0.04401 & -0.1179 & 185.375 & 3.175 & $0.04192-0.04670$ \\
HIP 981 & 0.41190 & -0.0227 & 309.498 & 0.612 & $0.41123-0.41257$ \\
G 108-21 & 0.50883 & -6.6740 & 17.660 & 178.558 & Not tested \\
HIP 3829 & 0.55083 & -0.2046 & 64.602 & 5.507 & $0.54261-0.55933$ \\
2MASS J07200325-0846499 & 0.64437 & -0.0152 & 240.683 & 0.411 & $0.64422-0.64460$ \\
HD 135226 & 0.90002 & -0.0953 & 62.902 & 2.567 & $0.89864-0.90144$ \\
HIP 21553 & 0.92358 & -0.4269 & 68.669 & 11.491 & $0.92010-0.92795$ \\
\hline
\end{tabular}

Notes. Only the results for HIP 981 are from a three-body calculation; see text for the explanation. The star HIP 21553 is additionally added since its minimum distance is only slightly over $1 \mathrm{pc}$ and it has a small relative velocity. The nominal orbit of 'Oumuamua and nominal stellar data are used for the results presented in Cols. 2-5. In the last column we show the minimum proximity distance variation interval obtained from the 10000 clones of 'Oumuamua.

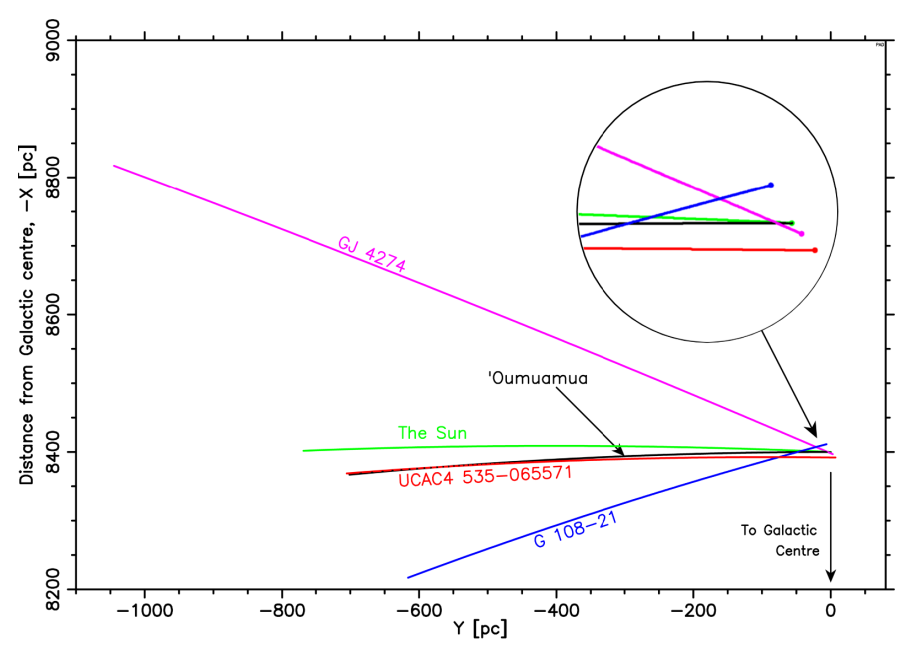

Fig. 1. Past trajectories of 'Oumuamua, the Sun, and three selected stars during the last $3 \mathrm{Myr}$. Their positions are projected onto the $X Y$ plane of the Galactocentric, non-rotating, right-handed rectangular frame. This plane coincides with the Galactic disk plane. The $O X$ axis is directed opposite to the Galactocentric direction to the Sun at the starting epoch $t=0$.

we restrict the error budget calculations to the influence of the 'Oumuamua orbit uncertainty.

To refine (and considerably shrink) our set of stellar perturbers, we first numerically followed the past motion of 'Oumuamua with each of the 201763 stars individually along with the Sun, forming a three-body problem under the influence of the full Galactic potential. During this preliminary calculation, we assumed all stellar masses to be $1.0 M_{\odot}$. Using these results, we selected 109 stellar objects that passed 'Oumuamua at a distance of closer than $3.5 \mathrm{pc}$. The parameters of all these encounters, derived from a nominal 'Oumuamua orbit and nominal stellar data, can be found in Table A.1.

After a detailed inspection involving the removal of obsolete objects and replacing components of multiple stellar systems with their respective centre of mass parameters, we finally collected a list of 57 stars or stellar systems which should be taken into account when studying 'Oumuamua's past motion in the solar neighbourhood. To use these stars as perturbers it was necessary to find estimations of their masses. It appeared that a lot of them are red (or even brown) dwarfs with a very small mass.
Additionally, we recognised several pairs of stars forming double systems as well as one triple system (Alpha Centauri A,B + Proxima) and calculated their centre of mass coordinates, total mass, and a systemic velocity. The most massive perturbers in our list are the Alpha Centauri and Sirius systems. A list of these perturbers with their estimated masses, starting positions and velocities is presented in Table A.2. In the last column of this table we present references for all values used by us. Some adopted mass values are rather crude estimations, but due to 'Oumuamua's large velocity it turned out that the change in a perturbers mass does not significantly influence the path of 'Oumuamua. This of course might be false for mutual interactions of perturbers.

Finally, we integrated the N-body problem, consisting of 'Oumuamua, the Sun, and all 57 individual stellar perturbers, a 59-body system under the influence of the overall Galactic potential (hereafter 59B model).

Figure 1 shows the past trajectories of 'Oumuamua (in black), the Sun (in green), and three example stars selected from Tables 3-4. Their motion is projected onto the Galactic disk plane.

\section{Results}

After analysing 'Oumuamua's past motion within the solar vicinity, we found seven encounters closer than 1 pc between 'Oumuamua and nearby stars. These encounters are described in Table 3 and presented in Fig. 2. The first 'Oumuamua star encounter (with HIP 3757) is a very close one, with a miss distance of only $0.04 \mathrm{pc}$ but with a rather high relative velocity of over $200 \mathrm{~km} \mathrm{~s}^{-1}$ taking place $118000 \mathrm{yr}$ ago. The second encounter, with GJ 4274, happened only 23000 yr ago with an even greater relative velocity of $316 \mathrm{~km} \mathrm{~s}^{-1}$. The minimum distance of the third event, with HIP 981, is also very close, but due to the large heliocentric distance of this event and practically unknown radial velocity of the star $\left(\mathrm{rv}=4.00 \pm 6.5 \mathrm{~km} \mathrm{~s}^{-1}\right.$, Barbier-Brossat \& Figon 2000) we treat this case as a "false positive". The remaining cases presented in Table 3 yield a relative velocity of over $60 \mathrm{~km} \mathrm{~s}^{-1}$, which also makes them not very promising candidates for 'Oumuamua's source system.

When searching for the parent star of 'Oumuamua, one probably should look for a close passage with a much smaller relative velocity. From among the over 200000 tested stars, we found only four such cases; see Table 4. 


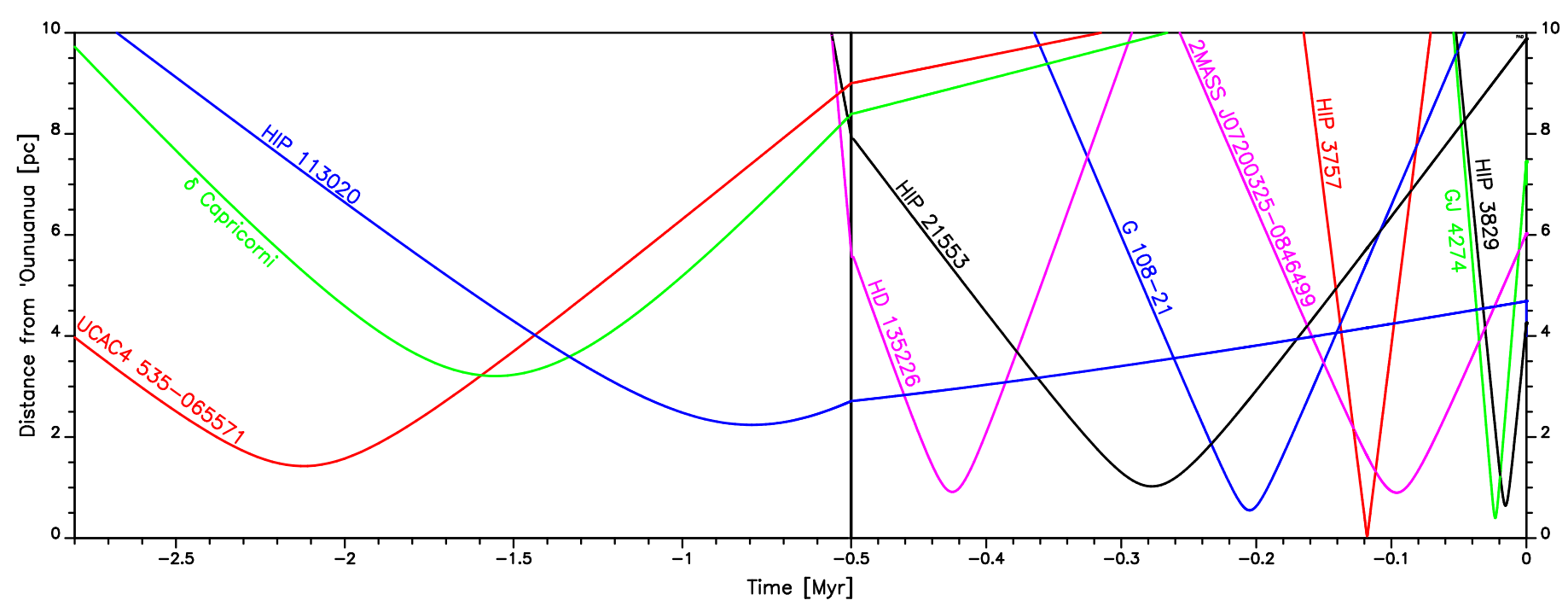

Fig. 2. Changes in the distance between 'Oumuamua and stars listed in Tables 3 and 4. Only HIP 981 and TYC 5325-1808-1 are omitted due to their unreliable kinematic data. Please note a horizontal scale change in the middle of the plot.

Table 4. Four cases of low-velocity encounters of 'Oumuamua with stars from our list.

\begin{tabular}{lrrrrc}
\hline \hline Star name & $\begin{array}{r}\text { Miss distance } \\
{[\mathrm{pc}]}\end{array}$ & $\begin{array}{r}\text { Epoch } \\
{[\mathrm{Myr}]}\end{array}$ & $\begin{array}{r}\text { Rel. velocity } \\
{\left[\mathrm{km} \mathrm{s}^{-1}\right]}\end{array}$ & $\begin{array}{r}r_{-} \text {hel } \\
{[\mathrm{pc}]}\end{array}$ & $\begin{array}{r}\text { Miss-distance interval } \\
{[\mathrm{pc}]}\end{array}$ \\
\hline TYC 5325-1808-1 & 2.93291 & -10.1215 & 7.940 & 269.220 & Not tested \\
UCAC4 535-065571 & 1.42718 & -2.1395 & 5.206 & 57.538 & $1.37201-1.48409$ \\
$\delta$ Capricorni & 3.21225 & -1.5259 & 6.872 & 41.049 & $3.16832-3.25921$ \\
HIP 113020 & 2.24104 & -0.7907 & 3.927 & 21.278 & $2.21875-2.26360$ \\
\hline
\end{tabular}

Notes. Columns 2-5 are from the 59B calculation. Only the results for TYC 5325-1808-1 are from the three-body integration; see text for the explanation. The last column presents the miss-distance variation interval from the 10000 clones of 'Oumuamua.

In Fig. 2 we show how the distance of 'Oumuamua from stars listed in Table 3 and Table 4 changed in time.

Almost $820000 \mathrm{yr}$ ago, 'Oumuamua passed near the star HIP 113020 (also known as BD-15 6290, GJ 876, or Ross 780) with a relative velocity of about $5 \mathrm{~km} \mathrm{~s}^{-1}$ and at a heliocentric distance of $21.3 \mathrm{pc}$. For the nominal 'Oumuamua orbit, the minimal distance between these two objects was 2.24 pc. However, it should be noted that (going backwards along its track) the motion of 'Oumuamua was perturbed by six stars from Table 3 as well as 51 other stars acting from larger distances of 1-3 pc. Every close passage of 'Oumuamua near a star magnifies starting point uncertainties, additionally increased by the stellar data errors. While most of the stars included in our calculations are M dwarfs with relatively small masses, some have masses greater than the Sun. However, while the stellar kinematics data uncertainties are the most important source of the proximity distance uncertainty, these are not estimated in this paper. To observe how the uncertainties of 'Oumuamua's orbit affect our results, we repeated our calculation of the 59B model for the 10000 clones of 'Oumuamua. We individually searched for the closest and the farthest clone at the approach epoch and recorded the encounter parameters for each star, obtaining their variation intervals. For this number of clones, these intervals are wider than $3 \sigma$ and are presented in the last column of Table 3. Similar data for all the studied stars may be found in Table A.3.

Three more low-velocity encounters happened further than $30 \mathrm{pc}$ from the Sun. We recognised the encounters with the high-proper-motion star UCAC4 535-065571 and the eclipsing binary $\delta$ Capricorni as the most interesting ones. TYC 53251808-1 cannot be reliably included in our list of perturbers since its mass and spectral type are unknown. The correct mass value is indispensable for dynamically tracing such a long trip (almost $270 \mathrm{pc})$.

UCAC4 535-065571 is a red dwarf of M6V spectral type and its mass is estimated to be $0.205 M_{\odot}$ (Newton et al. 2016) We obtained an encounter relative velocity of $5.35 \mathrm{~km} \mathrm{~s}^{-1}$ but with a minimum distance of $3.46 \mathrm{pc}$. With such a large miss-distance, one might reject this star as a parental candidate for 'Oumuamua. However, we noticed that the kinematics of UCAC4 535-065571 is rather poorly known. In the SIMBAD database we found its parallax, plx $=85.40 \pm 3.30$ mas (Dittmann et al. 2014), proper motions, pma $=-107.0 \pm 8$ mas $\mathrm{yr}^{-1}$ and $\mathrm{pmd}=-133.0 \pm 8 \mathrm{mas} \mathrm{yr}^{-1}$ (Zacharias et al. 2012), and radial velocity $\mathrm{rv}=-19 \pm 5 \mathrm{~km} \mathrm{~s}^{-1}$ Newton et al. (2014). By manipulating numbers within their uncertainties, we obtained a miss distance of $0.6 \mathrm{pc}$ but with a relative velocity of

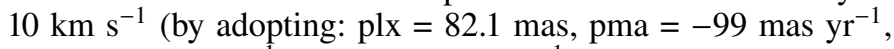

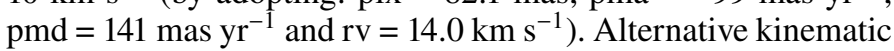
parameters for this star can also be found in West et al. (2015), where $\mathrm{plx}=76$ mas and $\mathrm{rv}=-9.5 \mathrm{~km} \mathrm{~s}^{-1}$. The discrepancy between radial-velocity measurements might be connected with the rotational velocity of $43 \mathrm{~km} \mathrm{~s}^{-1}$ (Newton et al. 2016) for this star. Using these kinematic data, we obtained a nominal proximity distance of $0.4 \mathrm{pc}$ but with a relative velocity of $14.7 \mathrm{~km} \mathrm{~s}^{-1}$.

$\delta$ Capricorni (HIP 107556, GJ 837) is also a good candidate, with high-precision kinematic parameters. It is an eclipsing 
binary so its mass is also accurate and it has a small relative velocity of $6.9 \mathrm{~km} \mathrm{~s}^{-1}$. 'Oumuamua passed this star at a rather large distance of $3.21 \mathrm{pc}$.

\section{Discussion and conclusions}

No obvious parent star has been identified. The closest 'Oumuamua - star proximity found by us, an encounter with HIP 3757 almost 120000 yr ago, does not indicate that 'Oumuamua originated from this star system; it might be true provided some mechanism of ejecting 'Oumuamua from this system with the relative velocity of $185 \mathrm{~km} \mathrm{~s}^{-1}$ be proposed.

It seems more reasonable to search for the parent star of 'Oumuamua in the cases of a much smaller relative velocity. Utilising such an approach would make HIP 113020 a more promising candidate. This well known star (known also as BD-15 6290, GJ 876, or Ross 780) has a rich planetary system consisting of four planets: one very small and three other massive and strongly interacting planets; see for example Rivera et al. (2010) and the references therein. Our resulting miss distance is highly sensitive to the systemic radial velocity of the HIP 113020 system. There seems to be some discrepancy between the centre of mass velocity of about $0.5 \mathrm{~km} \mathrm{~s}^{-1}$ obtained in the paper quoted above and the value presented in the SIMBAD database: $-1.519 \pm 0.157 \mathrm{~km} \mathrm{~s}^{-1}$ (Terrien et al. 2015). Taking into account that the motion of 'Oumuamua was perturbed by tens of stars after passing HIP 113020 and that kinematic parameters (and masses) of these perturbers are of a significantly different quality and accuracy, we cannot rule out the possibility that 'Oumuamua originated from the HIP 113020 planetary system. Definitively, we have to wait for much more precise stellar data from the Gaia mission (Gaia Collaboration 2016b).

In our results obtained for the vicinity of the Sun there is yet another nearby star worth mentioning. 'Oumuamua nominally passed HIP 21553 at a distance of 1.02287 pc almost $280000 \mathrm{yr}$ ago with a relative velocity of less than $35 \mathrm{~km} \mathrm{~s}^{-1}$. HIP 21553 (also known as HD 232979 or GJ 172) is a M0.5V-type red dwarf according to SIMBAD (Keenan \& McNeil 1989). Its astrometric data were recently highly improved by the Gaia mission (Gaia Collaboration 2016b).

Additionally, we found another candidate past the 30-pc heliocentric distance, the star UCAC4 535-065571. By varying this star's position and velocity within their respective uncertainty intervals, we obtained a very close encounter with 'Oumuamua at a reasonably small relative velocity of 5$15 \mathrm{~km} \mathrm{~s}^{-1}$. It seems necessary to study the kinematics of this star in more detail in order to make any definitive conclusion on the putative relation between this star and 'Oumuamua. There is also a small probability that 'Oumuamua comes from $\delta$ Capricorni.

An equally interesting hypothesis is that this interstellar object came to our planetary system from a more distant source.

Over the studied period of the past few million years, the heliocentric trajectory of 'Oumuamua appears to be almost a straight line with an approximately constant velocity. This is mainly because of its great velocity, relatively large distance from perturbing stars, and their small masses (in most cases).
This fact is illustrated in several figures included in Appendix B. One can also see in Fig. 1 that the deviation from the straight line motion is also very slight in the Galactocentric frame over a similar time interval.

Another important conclusion from our work is that despite the short observational interval of 'Oumuamua, its original orbit uncertainties do not influence our results in any significant way.

There recently appeared a preprint by S. Portegies Zwart and colleagues (Portegies Zwart et al. 2017) in which the authors propose five other stars as potential sources for 'Oumuamua. We carefully checked all these cases and according to our calculations 'Oumuamua did not come closer than $20 \mathrm{pc}$ to any of these stars. A probable reason for this finding is that an approximate dynamical model was used in the quoted paper, where as in all five cases these stars are very distant and therefore their motion is sensitive to the dynamical model details, especially the mutual interactions between all stars involved. Such a disagreement is also noted in Feng \& Jones (2018). On the contrary, these authors confirm our results for overlapping stars.

Acknowledgements. We would like to thank Ramon Brasser and the second anonymous referee for helpful comments and suggestions. This research was partially supported by the project 2015/17/B/ST9/01790 funded by the National Science Centre in Poland. This research made use of the SIMBAD database, operated at CDS, Strasbourg, France.

\section{References}

Barbier-Brossat, M., \& Figon, P. 2000, A\&A, 142, 217

Dittmann, J. A., Irwin, J. M., Charbonneau, D., \& Berta-Thompson, Z. K. 2014, ApJ, 784, 156

Drahus, M., Guzik, P., Waniak, W., et al. 2017, Nat. Astron., submitted [arXiv: 1712.00437]

Dybczyński, P. A., \& Berski, F. 2015, MNRAS, 449, 2459

Engelhardt, T., Jedicke, R., Vereš, P., et al. 2017, AJ, 153, 133

Feng, F., \& Jones, H. R. A. 2018, ApJ, 852, L27

Fraser, W. C., Pravec, P., Fitzsimmons, A., et al. 2017, ArXiv e-prints [arXiv: 1711.11530]

Gaia Collaboration (Brown, A. G. A., et al.) 2016a, A\&A, 595, A2

Gaia Collaboration (Prusti, T., et al.) 2016b, A\&A, 595, A1

Gaidos, E., Williams, J., \& Kraus, A. 2017, Res. Notes Am. Astron. Soc., 1, 13 Gontcharov, G. A. 2006, Astron. Lett., 32, 759

Irrgang, A., Wilcox, B., Tucker, E., \& Schiefelbein, L. 2013, A\&A, 549, A137

Jewitt, D., Luu, J., Rajagopal, J., et al. 2017, ApJ, 850, L36

Joshi, Y. C. 2007, MNRAS, 378, 768

Keenan, P. C., \& McNeil, R. C. 1989, ApJS, 71, 245

Kordopatis, G., \& RAVE Collaboration 2014, in Am. Astron. Soc. Meet. Abstr., 223, 346.02

Kunder, A., Kordopatis, G., Steinmetz, M., et al. 2017, AJ, 153, 75

Mamajek, E. 2017, Res. Notes Amer. Astron. Soc., 1, 21

Meech, K. J., Weryk, R., Micheli, M., et al. 2017, Nature, 552, 378

Newton, E. R., Charbonneau, D., Irwin, J., et al. 2014, AJ, 147, 20

Newton, E. R., Irwin, J., Charbonneau, D., et al. 2016, ApJ, 821, 93

Portegies Zwart, S., Pelupessy, I., Bedorf, J., Cai, M., \& Torres, S. 2017, ArXiv e-prints [arXiv: 1711.03558]

Rivera, E. J., Laughlin, G., Butler, R. P., et al. 2010, ApJ, 719, 890

Sitarski, G. 1998, Acta Astron., 48, 547

Terrien, R. C., Mahadevan, S., Bender, C. F., Deshpande, R., \& Robertson, P. 2015, ApJ, 802, L10

van Leeuwen F. 2007, A\&A, 474, 653

West, A. A., Weisenburger, K. L., Irwin, J., et al. 2015, ApJ, 812, 3

Zacharias, N., Finch, C. T., Girard, T. M., et al. 2012, VizieR Online Data Catalog: I/322 


\section{Appendix A: Supplementary material}

Table A.1. 109 individual objects selected form the SIMBAD data that fulfil the following conditions: an object must approach 'Oumuamua closer than $3.5 \mathrm{pc}$ before leaving the heliocentric sphere of $300 \mathrm{pc}$ radius.

\begin{tabular}{|c|c|c|c|c|c|}
\hline Star name & Alternative name & $\begin{array}{r}\text { Min. dist } \\
{[\mathrm{pc}]}\end{array}$ & $\begin{array}{l}\text { Epoch } \\
{[\mathrm{Myr}]}\end{array}$ & $\begin{array}{r}\text { Vrel } \\
{\left[\mathrm{km} \mathrm{s}^{-1}\right]} \\
\end{array}$ & $\begin{array}{r}r \text { hel } \\
\text { [pc] }\end{array}$ \\
\hline LP 646-17 & HIP 3757 & 0.04378 & -0.117935 & 207.96 & 3.175 \\
\hline GJ 4274 & LP $820-12$ & 0.41594 & -0.022642 & 316.28 & 0.611 \\
\hline HD 761 & HIP 981 & 0.50883 & -6.674040 & 17.66 & 178.558 \\
\hline GJ 3404 A & TYC 151-860-1 & 0.55191 & -0.204269 & 61.21 & 5.498 \\
\hline Wolf 28 & HIP 3829 & 0.65803 & -0.016006 & 266.49 & 0.432 \\
\hline 2MASS J07200325-0846499 & & 0.90252 & -0.094773 & 60.63 & 2.552 \\
\hline HD 135226 & BD-03 3748 & 0.91695 & -0.423887 & 73.23 & 11.408 \\
\hline HD 232979 & HIP 21553 & 1.03139 & -0.281024 & 34.65 & 7.564 \\
\hline TYC 5855-2215-1 & & 1.04275 & -6.751784 & 39.20 & 180.616 \\
\hline 2MASS J10433508+1213149 & & 1.15503 & -0.053937 & 266.61 & 1.453 \\
\hline$\alpha$ Centauri B & HIP 71681 & 1.25515 & 0.000000 & 35.39 & 0.001 \\
\hline Proxima Centauri & HIP 70890 & 1.30223 & 0.000000 & 37.09 & 0.001 \\
\hline$\alpha$ Centauri A & HIP 71683 & 1.32516 & 0.000000 & 35.03 & 0.001 \\
\hline$\alpha$ Centauri AB & & 1.34803 & 0.000000 & 36.57 & 0.001 \\
\hline UCAC4 535-065571 & & 1.42794 & -2.140431 & 5.36 & 57.564 \\
\hline GJ 358 & HIP 47425 & 1.54383 & -0.073742 & 122.26 & 1.986 \\
\hline GJ 793 & HIP 101180 & 1.67110 & -0.236802 & 32.72 & 6.374 \\
\hline Capella & HIP 24608 & 1.75796 & -0.485275 & 25.83 & 13.060 \\
\hline GJ 9603 & HIP 86916 & 1.79609 & -0.451911 & 43.43 & 12.163 \\
\hline Barnard's star & HIP 87937 & 1.82274 & 0.000000 & 134.83 & 0.001 \\
\hline GJ 4063 & TYC 3109-1699-1 & 1.82991 & -0.175405 & 40.07 & 4.722 \\
\hline GJ 195 A & Capella H & 1.84076 & -0.410834 & 32.61 & 11.057 \\
\hline HD 200325 & HIP 103749 & 1.85997 & -4.379190 & 12.06 & 117.531 \\
\hline G 208-45 & GJ $1245 \mathrm{~B}$ & 1.94326 & -0.119017 & 33.73 & 3.204 \\
\hline HZ 10 & WD $0407+179$ & 1.97167 & -0.545641 & 68.15 & 14.685 \\
\hline HD 8671 & HIP 6711 & 1.97438 & -1.106171 & 37.96 & 29.765 \\
\hline $\mathrm{BD}+31637$ & TYC 2355-291-1 & 2.02908 & -5.090162 & 26.82 & 136.492 \\
\hline GJ 9492 & HIP 71898 & 2.03615 & -0.282622 & 37.33 & 7.607 \\
\hline L 923-22 & GJ $754.1 \mathrm{~B}$ & 2.05851 & -0.176115 & 59.26 & 4.741 \\
\hline HD 24546 & HIP 18453 & 2.06574 & -0.907453 & 40.07 & 24.419 \\
\hline G 208-44 & GJ $1245 \mathrm{~A}$ & 2.07612 & -0.122176 & 31.54 & 3.289 \\
\hline LP $160-22$ & & 2.09918 & -0.526624 & 34.54 & 14.173 \\
\hline HIP 34603 & GJ 268 & 2.10932 & -0.188072 & 31.99 & 5.063 \\
\hline 2MASS J18212815+1414010 & & 2.11724 & -0.251783 & 35.39 & 6.777 \\
\hline GJ 65 B & & 2.15456 & -0.039837 & 34.10 & 1.073 \\
\hline GJ 909 A & HIP 117712 & 2.17334 & -0.521136 & 20.37 & 14.025 \\
\hline Wolf 359 & GJ 406 & 2.20907 & -0.027371 & 30.60 & 0.738 \\
\hline GJ 876 & HIP 113020 & 2.24392 & -0.817099 & 5.07 & 21.989 \\
\hline GJ 3376 A & HIP 28267 & 2.24657 & -0.198471 & 121.88 & 5.342 \\
\hline Kapteyn's star & HIP 24186 & 2.28553 & -0.010651 & 270.91 & 0.288 \\
\hline TYC 8470-213-1 & & 2.30049 & -0.172274 & 229.85 & 4.637 \\
\hline APMPM J0237-5928 & & 2.30106 & -0.097524 & 97.49 & 2.626 \\
\hline GJ 3287 & & 2.31517 & -1.072967 & 20.63 & 28.872 \\
\hline G 203-47 & HIP 83945 & 2.32770 & -0.093300 & 78.09 & 2.512 \\
\hline HD 91962 & HIP 51966 & 2.36104 & -3.529085 & 11.12 & 94.802 \\
\hline GJ 433.1 & HIP 56662 & 2.43579 & -0.126051 & 119.04 & 3.394 \\
\hline HD 175726 & HIP 92984 & 2.46005 & -0.772211 & 33.25 & 20.781 \\
\hline GJ 688 & HIP 86400 & 2.46025 & -0.255395 & 40.83 & 6.874 \\
\hline HD 162826 & HIP 87382 & 2.51056 & -1.196101 & 27.79 & 32.183 \\
\hline$\eta$ Casiopei B & GJ $34 \mathrm{~B}$ & 2.52756 & -0.205075 & 25.37 & 5.520 \\
\hline
\end{tabular}

Notes. Stars are sorted here by the proximity distance. To speed up the selection procedure, the motion of each object was traced individually as a three-body problem under the influence of the full Galactic potential. For its nominal orbit, we recorded the minimum 'Oumuamua - star distance (Min dist), moment of this approach (Epoch), relative velocity (Vrel), and heliocentric distance of the approach ( $r$ hel). A proximity epoch equalling zero means that the star's closest position was at the beginning of the backward numerical integration when 'Oumuamua was at 250 AU from the Sun. 
Table A.1. continued.

\begin{tabular}{|c|c|c|c|c|c|}
\hline Star name & Alternative name & $\begin{array}{r}\text { Min. dist } \\
{[\mathrm{pc}]}\end{array}$ & $\begin{array}{r}\text { Epoch } \\
{[\mathrm{Myr}]}\end{array}$ & $\begin{array}{r}\text { Vrel } \\
{\left[\mathrm{km} \mathrm{s}^{-1}\right]}\end{array}$ & $\begin{array}{r}r_{-} \text {hel } \\
{[\mathrm{pc}]}\end{array}$ \\
\hline HD 113376 & HIP 63797 & 2.52972 & -2.856293 & 40.47 & 76.776 \\
\hline GJ 1095 & HIP 35136 & 2.54526 & -0.197668 & 81.92 & 5.321 \\
\hline GJ 411 & HIP 54035 & 2.54679 & 0.000000 & 93.39 & 0.001 \\
\hline HD 201671 & HIP 104539 & 2.55447 & -10.508025 & 10.14 & 279.312 \\
\hline 2MASSI J1835379+325954 & & 2.58023 & -0.122667 & 39.52 & 3.302 \\
\hline 2MASS J05565722+1144333 & & 2.60458 & -0.085689 & 144.85 & 2.307 \\
\hline Aldebaran & HIP 21421 & 2.60688 & -0.492455 & 40.82 & 13.254 \\
\hline Sirius & HIP 32349 & 2.63821 & 0.000000 & 31.91 & 0.001 \\
\hline GJ 9105 C & HD $18143 \mathrm{C}$ & 2.67215 & -0.534272 & 28.67 & 14.379 \\
\hline GJ 725 B & HIP 91772 & 2.70964 & -0.058137 & 39.30 & 1.566 \\
\hline CD-56 1032 & HIP 22738 & 2.75803 & -0.613484 & 17.28 & 16.510 \\
\hline GJ $725 \mathrm{~A}$ & HIP 91768 & 2.77896 & -0.058064 & 36.82 & 1.564 \\
\hline GJ 54.1 & HIP 5643 & 2.79505 & -0.070215 & 32.52 & 1.891 \\
\hline [D75b] Star 1 & G $217-32$ & 2.82886 & -0.790870 & 17.31 & 21.283 \\
\hline GJ 752 & HIP 94761 & 2.83223 & -0.070143 & 66.46 & 1.889 \\
\hline GJ 15 A & HIP 1475 & 2.87070 & -0.048115 & 39.20 & 1.296 \\
\hline LP $412-31$ & & 2.87170 & -0.357225 & 38.54 & 9.615 \\
\hline GJ 644 & HIP 82817 & 2.87451 & -0.146364 & 36.53 & 3.940 \\
\hline TYC 7693-1161-1 & & 2.90315 & -0.734088 & 47.41 & 19.755 \\
\hline$\eta$ Casiopei & HIP 3821 & 2.90499 & -0.212720 & 23.37 & 5.726 \\
\hline GJ 729 & HIP 92403 & 2.91228 & -0.027389 & 21.15 & 0.738 \\
\hline GJ 15 B & HD 1326B & 2.91483 & -0.048154 & 38.84 & 1.297 \\
\hline TYC 5325-1808-1 & & 2.93291 & -10.121533 & 7.94 & 269.220 \\
\hline HD 317657 & TYC 7375-47-1 & 2.93350 & -0.408810 & 327.01 & 11.003 \\
\hline Teegarden's star & GAT 1370 & 2.94180 & -0.027297 & 86.90 & 0.736 \\
\hline GJ 376 B & HD $86728 B$ & 2.95912 & -0.255745 & 53.14 & 6.884 \\
\hline TYC 5172-2349-1 & & 3.00558 & -4.680653 & 35.14 & 125.576 \\
\hline GJ 702 & HIP 88601 & 3.01970 & -0.218690 & 19.04 & 5.887 \\
\hline Ruiz 207-61 & & 3.09432 & -0.146225 & 89.76 & 3.936 \\
\hline GJ 159.1 & HG 8-7 & 3.09673 & -0.329203 & 79.37 & 8.861 \\
\hline HD 18768 & HIP 14181 & 3.09713 & -0.474439 & 99.02 & 12.769 \\
\hline GJ 213 & HIP 26857 & 3.10918 & -0.048199 & 104.39 & 1.298 \\
\hline GJ 644 C & & 3.12047 & -0.147195 & 37.54 & 3.963 \\
\hline GJ 752 B & VB 10 & 3.12731 & -0.085166 & 61.00 & 2.293 \\
\hline GJ 905 & Ross 248 & 3.15691 & 0.000000 & 68.58 & 0.001 \\
\hline GJ 160.1 & HIP 19255 & 3.17841 & -0.872863 & 22.35 & 23.489 \\
\hline$\delta$ Capricorni & HIP 107556 & 3.20613 & -1.557216 & 7.20 & 41.893 \\
\hline HD 172051 & HIP 91438 & 3.21372 & -0.230299 & 52.90 & 6.199 \\
\hline$\epsilon$ Eridani & HIP 16537 & 3.21684 & 0.000000 & 33.32 & 0.001 \\
\hline GJ 701 & HIP 88574 & 3.24097 & -0.121593 & 58.79 & 3.274 \\
\hline GJ 643 & HIP 82809 & 3.25322 & -0.147410 & 37.72 & 3.968 \\
\hline GJ 887 & HIP 114046 & 3.26819 & -0.002325 & 93.26 & 0.064 \\
\hline LHS 1817 & LP 86-173 & 3.27213 & -0.097496 & 134.53 & 2.625 \\
\hline Wolf 1059 & LHS 5131 & 3.27918 & -0.090442 & 258.71 & 2.435 \\
\hline GJ 109 & HIP 12781 & 3.33010 & -0.212547 & 29.78 & 5.721 \\
\hline GJ 447 & HIP 57548 & 3.38087 & 0.000000 & 47.70 & 0.001 \\
\hline 2MASS J16452211-1319516 & & 3.38729 & -0.174101 & 45.74 & 4.687 \\
\hline HD 132730 & TYC 8681-841-1 & 3.39323 & -3.989147 & 15.80 & 107.110 \\
\hline GJ 866 & LP 820-64 & 3.40568 & 0.000000 & 78.04 & 0.001 \\
\hline GJ $745 \mathrm{~A}$ & HIP 93873 & 3.41696 & -0.121135 & 62.74 & 3.261 \\
\hline G 123-45 & & 3.42044 & -0.463265 & 22.31 & 12.468 \\
\hline HD 174153 & HIP 92519 & 3.43893 & -0.646559 & 77.15 & 17.400 \\
\hline GJ 3988 & LHS 3262 & 3.44475 & -0.119955 & 70.73 & 3.229 \\
\hline GJ 663 A & TYC 6820-326-1 & 3.44561 & -0.261330 & 14.76 & 7.034 \\
\hline GJ 745 B & HIP 93899 & 3.45640 & -0.121402 & 62.52 & 3.268 \\
\hline GJ 170 & LHS 1674 & 3.45761 & -0.403927 & 25.86 & 10.871 \\
\hline $\mathrm{BD}+50860 \mathrm{~B}$ & TYC 3339-1311-1 & 3.47850 & -1.406654 & 26.18 & 37.845 \\
\hline 61 Cygni A & HIP 104214 & 3.48546 & 0.000000 & 88.99 & 0.001 \\
\hline 61 Cygni B & HIP 104217 & 3.49692 & 0.000000 & 86.54 & 0.001 \\
\hline
\end{tabular}


Table A.2. Final list of 57 stars or star systems used as perturbers in our final dynamical model of 'Oumuamua's motion.

\begin{tabular}{|c|c|c|c|c|c|c|c|c|}
\hline Star name & $\begin{array}{l}\text { Mass } \\
{\left[M_{\odot}\right]} \\
\end{array}$ & $\begin{array}{c}X \\
{[\mathrm{pc}]}\end{array}$ & $\begin{array}{c}Y \\
{[\mathrm{pc}]}\end{array}$ & $\begin{array}{c}Z \\
{[\mathrm{pc}]}\end{array}$ & $\begin{array}{c}V \mathrm{x} \\
{\left[\mathrm{pc} \mathrm{Myr}^{-1}\right]}\end{array}$ & $\begin{array}{c}V y \\
{\left[\mathrm{pc} \mathrm{Myr}^{-1}\right]}\end{array}$ & $\begin{array}{c}V z \\
{\left[\mathrm{pc} \mathrm{Myr}^{-1}\right]}\end{array}$ & Ref. \\
\hline HIP 3757 & 0.4 & -4.869905 & 8.163363 & -23.203674 & -52.989230 & 46.668895 & -204.591445 & $1,1,1,2,3$ \\
\hline GJ 4274 & 0.14 & 3.223762 & 2.957145 & -6.039148 & 141.140365 & 94.217546 & -267.797888 & $4,5,6,2,7$ \\
\hline TYC 151-860-1 & 0.23 & -11.260623 & -6.162569 & -0.094524 & -66.113087 & -53.790236 & -5.867103 & $5,8,9,10,11$ \\
\hline HIP 3829, Wolf 28 & 0.68 & -1.210292 & 1.945959 & -3.594159 & -72.175698 & 69.395114 & -257.111463 & $12,12,12,13,14$ \\
\hline 2MASS J07200325-0846499 & 0.08 & -4.426203 & -4.074046 & 0.317199 & -60.908353 & -59.344984 & 1.872151 & $4,15,15,16,17$ \\
\hline TYC 5009-283-1 & 1.0 & 23.021112 & -1.443553 & 21.958699 & 43.238409 & -24.467213 & 42.944681 & $18,18,18,19,2$ \\
\hline HIP 21553 & 0.6 & -8.799950 & 4.438152 & 0.669586 & -43.309867 & -8.021714 & -1.980970 & $1,1,1,20,21$ \\
\hline 2MASS J10433508+1213149 & 0.08 & -4.833887 & -6.430379 & 12.182001 & -83.878243 & -136.754016 & 229.107657 & $4,22,22,22,23$ \\
\hline$\alpha$ Cent $\mathrm{AB}+$ Proxima system & 2.17 & 0.935517 & -0.893740 & 0.017400 & -29.182899 & 1.017810 & 12.535209 & $12,12,12+24,25+26+27,28$ \\
\hline UCAC4 535-065571 & 0.205 & 8.142407 & 6.867308 & 4.863749 & -8.368095 & -19.233016 & -5.616024 & $4,5,9,10,21$ \\
\hline HIP 47425 & 0.34 & -0.270092 & -9.499987 & 1.460452 & -33.489157 & -143.256893 & 18.063286 & $1,1,1,29,30$ \\
\hline HIP 101180 & 0.39 & -1.342284 & 7.662756 & 2.093016 & -20.645740 & 9.234058 & -5.321937 & $1,1,1,31,32$ \\
\hline HIP 24608 & 5.06 & -12.482300 & 3.914475 & 1.044814 & -36.754471 & -14.635561 & -9.310078 & $12,12,12,33,34$ \\
\hline HIP 86916 & 0.5 & 4.967614 & 16.744254 & 10.225582 & 2.385149 & 14.079262 & 12.221230 & \\
\hline TYC 3109-1699-1 & 0.2 & 2.479393 & 6.336940 & 2.491140 & 10.607473 & & & \\
\hline HIP 87937 & 0.16 & 1.516300 & 0.911399 & 0.443149 & -144.200647 & 4.701( & 18.610843 & $12,12,12,3$ \\
\hline HZ 10 & 1.0 & -34.764964 & 2.772228 & -15.508177 & -75.377293 & -14.473130 & -34.971842 & $5,5,39,40,42$ \\
\hline HIP 6711 & 1.39 & -25.879842 & 31.269062 & -13.945369 & -35.841132 & 5.440825 & -18.856965 & $1,1,1$, \\
\hline GJ 1245 ABC system & 0.28 & 0.868629 & 4.410703 & 0.672563 & 6.129215 & 4.675136 & -11.727727 & $45,10+46,47$ \\
\hline HIP 71898 & 0.48 & -2.134723 & 7.105483 & 8.076950 & -12.242474 & 1.061814 & 21.798225 & $1,1,1,3$ \\
\hline L 923-22 & 0.1 & 9.336853 & 5.513232 & -1.784881 & 38.014847 & 11.743817 & -7.341221 & $48,5,49,37,30$ \\
\hline HIP 34603 & 0.33 & -5.917432 & 0.108014 & 2.143010 & -44.405820 & -22.043392 & -7.659702 & $12,12,12,33,51$ \\
\hline GJ 65 AB & 0.21 & -0.697183 & 0.119872 & -2.527542 & -44.334435 & -18.602954 & -19.451010 & $52,52,52,53+54,55$ \\
\hline HIP 117712 & 1.09 & -5.173173 & 9.260300 & 2.469058 & -18.194848 & -4.381304 & -0.938894 & $12,12,12,13,56$ \\
\hline GJ 406 & 0.09 & -0.583077 & -1.198362 & 1.984705 & -28.448478 & -48.653982 & -13.935499 & $4,5,45,37,45$ \\
\hline HIP 28267 & 1.3 & -20.646959 & -11.843636 & -5.640298 & -110.785271 & -92.744647 & -36.941301 & $1,1,1,31,57$ \\
\hline HIP 113020 & 0.334 & 1.441102 & 1.905640 & -4.034279 & -12.583839 & -20.164310 & -12.213344 & $12,12,12,37,58$ \\
\hline TYC 8470-213-1 & 1.0 & 15.179662 & -15.745125 & -34.620438 & 82.441710 & -102.422426 & -208.063306 & $1,1,1,2,2$ \\
\hline HIP 24186 & 0.39 & -1.057028 & -2.982685 & -2.299061 & 19.991822 & -294.248666 & -54.060419 & 12,12 \\
\hline I J0237-5928 & 0.22 & 0.989962 & -5.626783 & -7.766258 & -23.371954 & -73.481326 & -93.0 & \\
\hline HIP 83945 & 0.266 & 2.166005 & 5.597234 & 4.406165 & 26.0 & 9697 & 19.6 & 12,12, \\
\hline HIP 56662 & 0.935 & -4.247950 & -1.484937 & 15.153736 & -41.484216 & -15.924967 & 109.922558 & 12,12 \\
\hline HIP 86400 & 0.85 & 9.274425 & 4.883523 & 3.336740 & 17.935902 & -0.693717 & 11.375820 & $12,12,12,19,65$ \\
\hline HIP 35136 & 0.9 & -15.269627 & 2.598365 & 6.739659 & -81.650214 & -1.684617 & 33.045457 & $12,12,12,31,66$ \\
\hline HIP 54035 & 0.46 & -1.054711 & -0.094472 & 2.316283 & 47.197616 & -54.908745 & -75.981741 & $12,12,12,31,67$ \\
\hline 2MASSI J1835379+325954 & 0.07 & 2.435869 & 4.513765 & 1.611691 & 21.323286 & -0.059501 & -3.403988 & $4,68,9,69,9$ \\
\hline 2MASS J05565722+1144333 & 0.15 & -12.556104 & -3.645129 & -1.479015 & -148.572944 & -78.990706 & 0.269334 & $4,44,9,70,71$ \\
\hline HIP 32349 & 2.99 & -1.815217 & -1.874932 & -0.379090 & 13.087100 & -2.296652 & -12.179029 & $12,12,12,13,72$ \\
\hline GJ 725 AB system & 0.58 & 0.039863 & 3.202252 & 1.441291 & -25.325856 & -11.833516 & 26.619384 & $1,1,1,31,47$ \\
\hline HIP 22738 & 0.7 & -1.192678 & -8.579194 & -6.955349 & -9.646365 & -35.215095 & -20.407536 & $12,12,12,29,50$ \\
\hline HIP 5643 & 0.13 & -0.692360 & 0.466957 & -3.594156 & -29.284995 & 0.424845 & -23.796103 & $12,12,12,31,30$ \\
\hline GJ 752 AB system & 0.55 & 4.481805 & 3.820433 & -0.338091 & 53.884411 & -8.729523 & & $4+1,73+1,73+1,10+20,47$ \\
\hline GJ 15 AB system & 0.6 & -1.51 & & -1.1285 & -50.153 & -12.74 & -3.66 & $1+4,1+44,1+74,3$ \\
\hline HIP 92403 & 0.17 & 2.855273 & 0.650863 & -0.493625 & -12.182465 & -1.279999 & -7.549572 & $12,12,12,31,21$ \\
\hline Teegarden's star & 0.08 & -2.883244 & 1.034454 & -2.310472 & -66.768384 & -73.067253 & -56.387473 & $4,44,61,10,21$ \\
\hline HIP 88601 & 1.62 & 4.320600 & 2.483796 & 1.001895 & 6.169135 & -19.189299 & -14.679349 & $12,12,12,75,76$ \\
\hline Ruiz 207-61 & 0.08 & -2.466202 & -13.392706 & -4.316815 & -44.855241 & -106.443188 & -26.577086 & $4,68,45,77,78$ \\
\hline HIP 26857 & 0.18 & -5.590121 & -1.363571 & -0.933193 & -91.980164 & -91.361241 & 8.418275 & $12,12,12,79,47$ \\
\hline Ross 248 & 0.12 & -1.032642 & 2.838561 & -0.920011 & 33.153204 & -76.262790 & 0.284634 & $4,80,80,37,21$ \\
\hline HIP 91438 & 0.9 & 12.669348 & 2.882727 & -1.537885 & 38.206785 & -2.328500 & -4.403618 & $12,12,12,19,81$ \\
\hline$\delta$ Capricorni & 2.06 & 6.511185 & 5.075845 & -8.523903 & -7.436483 & -18.343120 & -11.762829 & $12,12,12,33,82$ \\
\hline HIP 16537 & 0.85 & -2.098618 & -0.549497 & -2.374208 & -3.862226 & 7.417522 & -21.063553 & $12,12,12,31,83$ \\
\hline HIP 88574 & 0.5 & 6.950725 & 3.231218 & 1.200757 & 33.830913 & 14.644326 & -19.358860 & $12,12,12,31,30$ \\
\hline HIP 114046 & 0.53 & 1.299173 & 0.211052 & -2.999850 & -96.274433 & -13.614020 & -51.028239 & $12,12,12,84,30$ \\
\hline HIP 57548 & 0.16 & 0.004397 & -1.712804 & 2.914646 & 18.364701 & 5.194591 & -33.949087 & $1,1,1,37,21$ \\
\hline GJ 866 ABC system & 0.30 & 1.249826 & 1.390358 & -2.847040 & -69.843346 & -1.090148 & 42.215336 & $4,85,86,25,87$ \\
\hline 61 Cyg AB system & 1.33 & 0.464345 & 3.442752 & -0.354214 & -95.544501 & -55.395340 & -8.848684 & $12,12,12,31,88$ \\
\hline
\end{tabular}

Notes. Heliocentric Galactic velocities are in parsec per Myr. To obtain velocities in $\mathrm{km} \mathrm{s}^{-1}$, each component must be divided by 1.0227 . Stars are presented here in the same sequence as in Table A.3. In the last column we include references for all data used to produce this table. For each star or stellar system we present sources of: positions, proper motions, parallaxes, radial velocities and masses in this order. In some cases of multiple systems we present individual member references connected with a plus sign. 


\section{References for Table A.2}

1. Gaia Collaboration (Brown, A. G. A, et al.) 2016a, A\&A, 595, A2

2. Kordopatis, G., Gilmore, G., Steinmetz, M., et al. 2013, AJ, 146, 134

3. Gaidos, E., Mann, A. W., Lépine, S., et al. 2014, MNRAS, 443, 2561

4. Cutri, R. M., Skrutskie, M. F., van Dyk, S., et al. 2003, VizieR Online Data Catalog: II/246

5. Zacharias, N., Finch, C. T., Girard, T. M., et al. 2012, VizieR Online Data Catalog: I/322

6. Bobylev, V. V. 2017, Astron. Rep., 61, 883

7. Bonfils, X., Delfosse, X., Udry, S., et al. 2013, A\&A, 549, A109

8. Høg, E., Fabricius, C., Makarov, V. V., et al. 2000, A\&A, 355, L27

9. Dittmann, J. A., Irwin, J. M., Charbonneau, D., \& Berta-Thompson, Z. K. 2014, ApJ, 784, 156

10. Newton, E. R., Charbonneau, D., Irwin, J., et al. 2014, AJ, 147, 20

11. Neves, V., Bonfils, X., Santos, N. C., et al. 2013, A\&A, 551, A36

12. van Leeuwen, F. 2007, A\&A, 474, 653

13. Gontcharov, G. A. 2006, Astron. Lett., 32, 759

14. Giammichele, N., Bergeron, P., \& Dufour, P. 2012, ApJS, 199, 29

15. Faherty, J. K., Burgasser, A. J., Walter, F. M., et al. 2012, ApJ, 752,56

16. Adelman-McCarthy, J. K., et al. 2009, VizieR Online Data Catalog: II $/ 294$

17. Huber, D., Bryson, S. T., et al. 2017, VizieR Online Data Catalog: IV/034

18. Gaia Collaboration (Prusti, T., et al.) 2016b, A\&A, 595, A1

19. Kunder, A., Kordopatis, G., Steinmetz, M., et al. 2017, AJ, 153, 75

20. Soubiran, C., Jasniewicz, G., Chemin, L., et al. 2013, A\&A, 552, A64

21. Newton, E. R., Irwin, J., Charbonneau, D., et al. 2017, ApJ, 834, 85

22. Burgasser, A. J., Gillon, M., Melis, C., et al. 2015a, AJ, 149, 104

23. Burgasser, A. J., Melis, C., Todd, J., et al. 2015c, AJ, 150, 180

24. Dupuy, T. J., \& Liu, M. C. 2012, ApJS, 201, 19

25. Wilson, R. E. 1953, Carnegie Institute, Washington, DC, Publication

26. Valenti, J. A., \& Fischer, D. A. 2005, ApJS, 159, 141

27. Torres, C. A. O., Quast, G. R., da Silva, L., et al. 2006, A\&A, 460, 695

28. Kervella, P., Thévenin, F., \& Lovis, C. 2017, A\&A, 598, L7

29. García-Sánchez, J., Weissman, P. R., Preston, R. A., et al. 2001, A\&A, 379, 634

30. Astudillo-Defru, N., Delfosse, X., Bonfils, X., et al. 2017, A\&A, 600, A13

31. Nidever, D. L., Marcy, G. W., Butler, R. P., Fischer, D. A., \& Vogt, S. S. 2002, ApJS, 141, 503

32. Perger, M., García-Piquer, A., Ribas, I., et al. 2017, A\&A, 598, A26

33. Karataş, Y., Bilir, S., Eker, Z., \& Demircan, O. 2004, MNRAS, 349, 1069

34. Torres, G., Claret, A., Pavlovski, K., \& Dotter, A. 2015, ApJ, 807, 26

35. Kharchenko, N. V., Scholz, R.-D., Piskunov, A. E., Röser, S., \& Schilbach, E. 2007, Astron. Nachr., 328, 889

36. Reid, I. N., \& Cruz, K. L. 2002, AJ, 123, 2806

37. Terrien, R. C., Mahadevan, S., Bender, C. F., Deshpande, R., \& Robertson, P. 2015, ApJ, 802, L10

38. Gaidos, E., \& Mann, A. W. 2014, ApJ, 791, 54

39. van Altena, W. F., Lee, J. T., \& Hoffleit, E. D. 1995, The General Catalogue of Trigonometric [Stellar] Parallaxes (New Haven, CT: Yale University Observatory)

40. Greenstein, J. L., \& Trimble, V. L. 1967, ApJ, 149, 283

41. Muirhead, P. S., Johnson, J. A., Apps, K., et al. 2012, ApJ, 747, 144

42. Guo, J., Zhao, J., Tziamtzis, A., et al. 2015, MNRAS, 454, 2787

43. Ramírez, I., Fish, J. R., Lambert, D. L., \& Allende Prieto, C. 2012, ApJ, 756, 46

44. Lépine, S., \& Shara, M. M. 2005, AJ, 129, 1483

45. Jenkins, J. S., Ramsey, L. W., Jones, H. R. A., et al. 2009, ApJ, 704, 975

46. Morin, J., Donati, J.-F., Petit, P., et al. 2010, MNRAS, 407, 2269
47. Mann, A. W., Feiden, G. A., Gaidos, E., Boyajian, T., \& von Braun, K. 2015, ApJ, 804, 64

48. Zacharias, N., Urban, S. E., Zacharias, M. I., et al. 2003, VizieR Online Data Catalog: I/289

49. Harrington, R. S., \& Dahn, C. C. 1980, AJ, 85, 454

50. Ward-Duong, K., Patience, J., De Rosa, R. J., et al. 2015, MNRAS, 449, 2618

51. Barry, R. K., Demory, B.-O., Ségransan, D., et al. 2012, ApJ, 760, 55

52. Winters, J. G., Henry, T. J., Lurie, J. C., et al. 2015, AJ, 149, 5

53. Montes, D., López-Santiago, J., Gálvez, M. C., et al. 2001, MNRAS, 328,45

54. Benedict, G. F., Henry, T. J., Franz, O. G., et al. 2016, AJ, 152, 141

55. Kervella, P., Mérand, A., Ledoux, C., Demory, B.-O., \& Le Bouquin, J.-B. 2016, A\&A, 593, A127

56. Agati, J.-L., Bonneau, D., Jorissen, A., et al. 2015, A\&A, 574, A6

57. Eggenberger, A., Udry, S., Chauvin, G., et al. 2007, A\&A, 474, 273

58. Correia, A. C. M., Couetdic, J., Laskar, J., et al. 2010, A\&A, 511, A21

59. Anglada-Escudé, G., Arriagada, P., Tuomi, M., et al. 2014, MNRAS, 443, L89

60. Scholz, R.-D., Irwin, M., Ibata, R., Jahreiß, H., \& Malkov, O. Y. 2000, A\&A, 353, 958

61. Henry, T. J., Jao, W.-C., Subasavage, J. P., et al. 2006, AJ, 132, 2360

62. Scholz, R.-D., Irwin, M., Schweitzer, A., \& Ibata, R. 1999, A\&A, 345, L55

63. Zhang, Y.-Y., Deng, L.-C., Liu, C., et al. 2013, AJ, 146, 34

64. Tremblay, P.-E., Gentile-Fusillo, N., Raddi, R., et al. 2017, MNRAS, 465, 2849

65. Katoh, N., Itoh, Y., Toyota, E., \& Sato, B. 2013, AJ, 145, 41

66. Casagrande, L., Schönrich, R., Asplund, M., et al. 2011, A\&A, 530, A138

67. Ségransan, D., Kervella, P., Forveille, T., \& Queloz, D. 2003, A\&A, 397, L5

68. Schmidt, S. J., Cruz, K. L., Bongiorno, B. J., Liebert, J., \& Reid, I. N. 2007, AJ, 133, 2258

69. Deshpande, R., Martín, E. L., Montgomery, M. M., et al. 2012, AJ, 144, 99

70. Lépine, S., Rich, R. M., \& Shara, M. M. 2003, AJ, 125, 1598

71. Newton, E. R., Irwin, J., Charbonneau, D., et al. 2016, ApJ, 821, 93

72. Bond, H. E., Schaefer, G. H., Gilliland, R. L., et al. 2017, ApJ, 840,70

73. Monet, D. G., Dahn, C. C., Vrba, F. J., et al. 1992, AJ, 103, 638

74. Dittmann, J. A., Irwin, J. M., Charbonneau, D., \& Berta-Thompson, Z. K. 2014, ApJ, 784, 156

75. Pourbaix, D., Tokovinin, A. A., Batten, A. H., et al. 2004, A\&A, 424, 727

76. Fernandes, J., Lebreton, Y., Baglin, A., \& Morel, P. 1998, A\&A, 338, 455

77. Burgasser, A. J., Logsdon, S. E., Gagné, J., et al. 2015b, ApJS, 220, 18

78. Filippazzo, J. C., Rice, E. L., Faherty, J., et al. 2015, ApJ, 810, 158

79. Chubak, C., \& Marcy, G. 2011, in Am. Astron. Soc. Meet. Abstr. \#217, Bull. Am. Astron. Soc., 43, 434.12

80. Gatewood, G. 2008, AJ, 136, 452

81. Fuhrmann, K., Chini, R., Kaderhandt, L., \& Chen, Z. 2017, ApJ, 836, 139

82. Thalmann, C., Desidera, S., Bonavita, M., et al. 2014, A\&A, 572, A91

83. Pinheiro, F. J. G., Fernandes, J. M., Cunha, M. S., et al. 2014, MNRAS, 445, 2223

84. Desidera, S., Covino, E., Messina, S., et al. 2015, A\&A, 573, A126

85. Salim, S., \& Gould, A. 2003, ApJ, 582, 1011

86. Torres, G., Andersen, J., \& Giménez, A. 2010, A\&ARv, 18, 67

87. Ségransan, D., Kervella, P., Forveille, T., \& Queloz, D. 2003, A\&A, 397, L5

88. Kervella, P., Mérand, A., Pichon, B., et al. 2008, A\&A, 488, 667 
A\&A 610, L11 (2018)

Table A.3. Minimal distances between the 10000 clones of 'Oumuamua and 57 stars included in our research.

\begin{tabular}{|c|c|c|c|c|}
\hline Star name & Min. dist [pc] & Epoch [Myr] & Rel. velocity $\left[\mathrm{km} \mathrm{s}^{-1}\right]$ & dist_hel [pc] \\
\hline HIP 3757 & $0.042: 0.044: 0.047$ & $0.1179: 0.1179: 0.1180$ & $208.00: 207.96: 207.92$ & $3.17: 3.17: 3.18$ \\
\hline GJ 4274 & $0.411: 0.412: 0.413$ & $0.0227: 0.0227: 0.0227$ & $316.28: 316.27: 316.27$ & $0.61: 0.61: 0.61$ \\
\hline TYC $151-860-1$ & $0.543: 0.551: 0.559$ & $0.2044: 0.2046: 0.2047$ & $61.24: 61.21: 61.17$ & $5.50: 5.51: 5.52$ \\
\hline HIP 3829, Wolf 28 & $0.644: 0.644: 0.645$ & $0.0152: 0.0152: 0.0152$ & $266.50: 266.49: 266.52$ & $0.41: 0.41: 0.41$ \\
\hline 2MASS J07200325-0846499 & $0.899: 0.900: 0.901$ & $0.0953: 0.0953: 0.0954$ & $60.65: 60.63: 60.60$ & $2.56: 2.57: 2.57$ \\
\hline TYC 5009-283-1 & $0.920: 0.924: 0.928$ & $0.4271: 0.4270: 0.4268$ & $73.18: 73.23: 73.29$ & $11.48: 11.49: 11.50$ \\
\hline HIP 21553 & $1.013: 1.023: 1.033$ & $0.2772: 0.2774: 0.2778$ & $34.67: 34.65: 34.62$ & $7.45: 7.47: 7.49$ \\
\hline 2MASS J10433508+1213149 & $1.154: 1.157: 1.160$ & $0.0540: 0.0540: 0.0540$ & $266.60: 266.62: 266.63$ & $1.45: 1.45: 1.46$ \\
\hline$\alpha$ Cen AB + Proxima system & $1.294: 1.294: 1.294$ & $0.0000: 0.0000: 0.0000$ & $35.27: 35.27: 35.27$ & $0.00: 0.00: 0.00$ \\
\hline UCAC4 535-065571 & $1.372: 1.427: 1.484$ & $2.1292: 2.1395: 2.1490$ & $5.40: 5.36: 5.32$ & $57.33: 57.54: 57.73$ \\
\hline HIP 47425 & $1.517: 1.519: 1.521$ & $0.0758: 0.0758: 0.0758$ & $122.26: 122.26: 122.26$ & $2.04: 2.04: 2.04$ \\
\hline HIP 101180 & $1.659: 1.671: 1.683$ & $0.2346: 0.2345: 0.2344$ & $32.71: 32.72: 32.72$ & $6.32: 6.31: 6.30$ \\
\hline HIP 24608 & $1.732: 1.748: 1.764$ & $0.4927: 0.4927: 0.4928$ & $25.79: 25.83: 25.87$ & $13.28: 13.26: 13.25$ \\
\hline & $1.790: 1.794: 1.799$ & $0.4533: 0.4533: 0.45$ & $43.40: 43.43: 43$ & $12.19: 12.20: 12.21$ \\
\hline TYC 3109-1699-1 & $1.820: 1.822: 1.823$ & $0.1705: 0.1$ & $7: 40.10$ & $4.58: 4$ \\
\hline HIP 87937 & $1.823: 1.823: 1.823$ & $0.0000: 0.0$ & $3: 134.86$ & $0.00: 0$ \\
\hline HZ 10 & $1.964: 1.975: 1.986$ & $0.5450: 0.5449: 0.5447$ & $68.20: 68.15: 68.10$ & $14.65: 14.66: 14.68$ \\
\hline HIP 6711 & $1.958: 1.976: 1.995$ & $1.1063: 1.1068: 1.1074$ & $37.99: 37.96: 37.93$ & $29.73: 29.78: 29.83$ \\
\hline GJ 1245 ABC system & $2.023: 2.024: 2.024$ & $0.1238: 0.1225: 0.1229$ & $32.31: 32.33: 32.35$ & $3.33: 3.30: 3.31$ \\
\hline HIP 71898 & $2.025: 2.038: 2.052$ & $0.2844: 0.2845: 0.2848$ & $37.30: 37.33: 37.36$ & $7.64: 7.66: 7.67$ \\
\hline L 923-22 & $2.051: 2.056: 2.060$ & $0.1790: 0.1769: 0.1771$ & $59.23: 59.26: 59.30$ & $4.81: 4.76: 4.77$ \\
\hline HIP 34603 & $2.091: 2.098: 2.105$ & $0.1819: 0.18$ & $31.95: 31.9$ & $4.90: 4.89: 4.88$ \\
\hline GJ $65 \mathrm{AB}$ & $2.151: 2.152: 2.153$ & $0.0438: 0.0$ & 34. & $: 1.18$ \\
\hline HIP & $2.138: 2.166: 2.194$ & & 20 & $2: 13.83$ \\
\hline & $2.207: 2.208: 2.209$ & & 30. & \\
\hline HIP & $2.211: 2$ & & $8: 121.84$ & $6: 5.28$ \\
\hline HIP 11 & $2.219: 2.241: 2.264$ & $0.7902: 0.7$ & $5.04: 5.07: 5.11$ & $21.29: 21.28: 21.28$ \\
\hline TYC 8470-213-1 & $2.263: 2.268: 2.276$ & $0.1738: 0.1743: 0.1746$ & $229.86: 229.85: 229.83$ & $4.67: 4.69: 4.71$ \\
\hline HIP 24186 & $2.273: 2.274: 2.274$ & $0.0115: 0.0115: 0.01$ & $270.91: 270.91: 270.90$ & $0.31: 0.31: 0.31$ \\
\hline APMPM J0237-5928 & $2.274: 2.278: 2.282$ & $0.0954: 0.0953: 0.0953$ & $97.45: 97.49: 97.52$ & $2.57: 2.57: 2.56$ \\
\hline HIP 83945 & $2.297: 2.298: 2.299$ & $0.0889: 0.0888: 0.0888$ & $78.12: 78.09: 78.05$ & $2.40: 2.39: 2.39$ \\
\hline HIP 56662 & $2.419: 2.421: 2.425$ & $0.1282: 0.1288: 0.1293$ & $119.01: 119.04: 119.06$ & : 3.49 \\
\hline & $2.459: 2.461: 2.463$ & & $40.88: 40$. & 6.84 \\
\hline & 51 & & & $: 5.38$ \\
\hline $\mathrm{HIF}$ & $54: 2$ & 000 & 93 & $: 0.00$ \\
\hline $2 \mathrm{M}$ & $2.569: 2.569: 2.570$ & 0.1162 & $2: 39.55$ & $6: 3.13$ \\
\hline S J05565722+1144333 & $2.605: 2.608: 2.612$ & $4: 0.0854$ & $144.89: 144.85: 144.81$ & $0: 2.30$ \\
\hline HIP 32349 & $2.638: 2.638: 2.638$ & $0.0000: 0.00$ & $31.89: 31.91: 31.94$ & $0: 0.00$ \\
\hline GJ 725 AB system & $2.747: 2.748: 2.748$ & $0.0573: 0.0573: 0.0573$ & $37.89: 37.87: 37.85$ & $1.54: 1.54: 1.54$ \\
\hline HIP 22738 & $2.740: 2.758: 2.776$ & $0.6045: 0.6042: 0.6145$ & $17.30: 17.28: 17.26$ & $16.25: 16.26: 16.56$ \\
\hline HIP 5643 & $2.792: 2.794: 2.796$ & $0.0721: 0.0721: 0.0721$ & $32.55: 32.52: 32.48$ & $1.94: 1.94: 1.95$ \\
\hline GJ $752 \mathrm{AB}$ system & $2.840: 2.841: 2.842$ & $0.0775: 0.07$ & $65.63: 65.67: 65.71$ & $2.08: 2.09: 2.09$ \\
\hline GJ 1 & $2.875: 2.87$ & & 39. & $2: 1.42$ \\
\hline HIP & $2.911: 2.912: 2.913$ & $0.0270: 0.0270: 0.0262$ & $6: 21.16: 21.15$ & $3: 0.71$ \\
\hline rden's star & $2.941: 2.942: 2.942$ & $0.0277: 0.0277: 0.0277$ & $86.95: 86.90: 86.85$ & $0.75: 0.75: 0.75$ \\
\hline HIP 88601 & $3.007: 3.015: 3.023$ & $0.2105: 0.2103: 0.2102$ & $19.06: 19.04: 19.01$ & $5.67: 5.66: 5.65$ \\
\hline Ruiz 207-61 & $3.047: 3.052: 3.052$ & $0.1509: 0.1538: 0.1537$ & $89.73: 89.76: 89.77$ & $4.07: 4.14: 4.14$ \\
\hline HIP 26857 & $3.101: 3.103: 3.105$ & $0.0457: 0.0458: 0.0458$ & $104.41: 104.39: 104.36$ & $1.23: 1.23: 1.24$ \\
\hline Ross 248 & $3.157: 3.157: 3.157$ & $0.0000: 0.0000: 0.0000$ & $68.56: 68.58: 68.61$ & $0.00: 0.00: 0.00$ \\
\hline HIP 91438 & $3.202: 3.206: 3.210$ & $0.2344: 0.2345: 0.2346$ & $52.86: 52.90: 52.94$ & $6.30: 6.31: 6.32$ \\
\hline$\delta$ Capricorni & $3.168: 3.212: 3.259$ & $1.5214: 1.5259: 1.5317$ & $7.20: 7.20: 7.21$ & $40.88: 41.05: 41.26$ \\
\hline HIP 16537 & $3.217: 3.217: 3.217$ & $0.0000: 0.0000: 0.0000$ & $33.32: 33.32: 33.32$ & $0.00: 0.00: 0.00$ \\
\hline HIP 88574 & $3.225: 3.231: 3.236$ & $0.1173: 0.1173: 0.1173$ & $58.81: 58.79: 58.76$ & $3.16: 3.16: 3.15$ \\
\hline HIP 114046 & $3.268: 3.268: 3.268$ & $0.0023: 0.0023: 0.0023$ & $93.31: 93.26: 93.21$ & $0.06: 0.06: 0.06$ \\
\hline HIP 57548 & $3.381: 3.381: 3.381$ & $0.0000: 0.0000: 0.0000$ & $47.71: 47.70: 47.70$ & $0.00: 0.00: 0.00$ \\
\hline GJ 866 ABC system & $3.406: 3.406: 3.406$ & $0.0000: 0.0000: 0.0000$ & $78.05: 78.04: 78.04$ & $0.00: 0.00: 0.00$ \\
\hline 61 Cyg AB system & $3.491: 3.491: 3.491$ & $0.0000: 0.0000: 0.0000$ & $87.79: 87.83: 87.86$ & $0.00: 0.00: 0.00$ \\
\hline
\end{tabular}

Notes. Here we present variation intervals roughly equivalent to the $\pm 4 \sigma$ deviations, obtained from among the 10000 encounters. In the last column we present a heliocentric distance for this event. Epochs are in the past, but minuses are omitted. Proximity epochs equal to zero mean that the star's closest position is at the beginning of the backward numerical integration (but they are still acting as perturbers). 


\section{Appendix B: Geometry examples of 'Oumuamua encounters with selected stars}

Below we present six plots of the example geometries of the close 'Oumuamua - star passages. We use here a heliocentric, non-rotating, right-handed rectangular frame. The $X Y$ plane is parallel to the Galactic disk plane and the $O X$ axis is directed to the Galactic Centre at the beginning of the calculation. Green lines depict the 'Oumuamua motion while the red ones show the star trajectory. Open circles mark the starting positions of 'Oumuamua and the star.

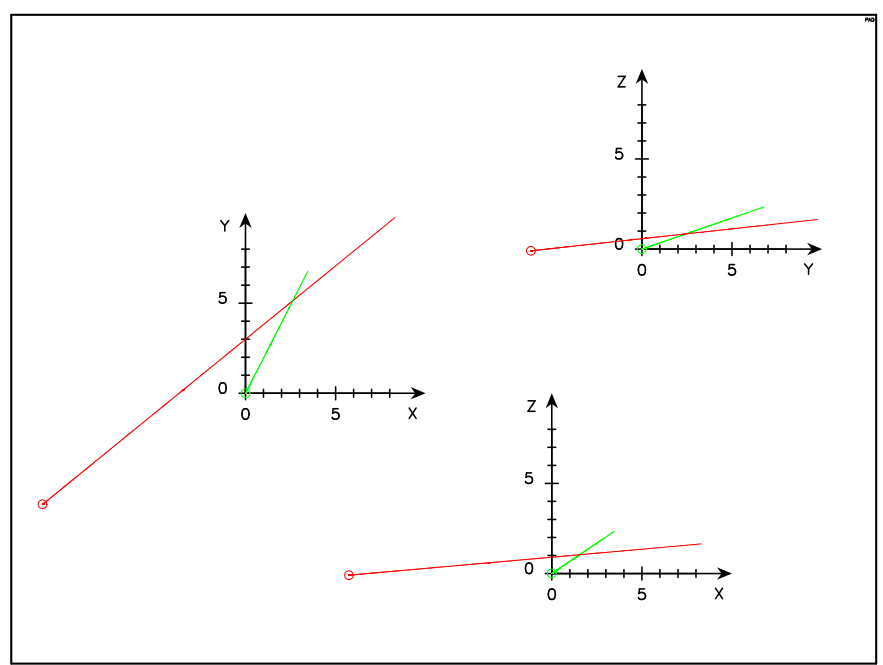

Fig. B.1. Geometry of the encounter of 'Oumuamua with the star G 108-21 0.2 Myr ago. Depicted is 0.3 Myr of their motion.

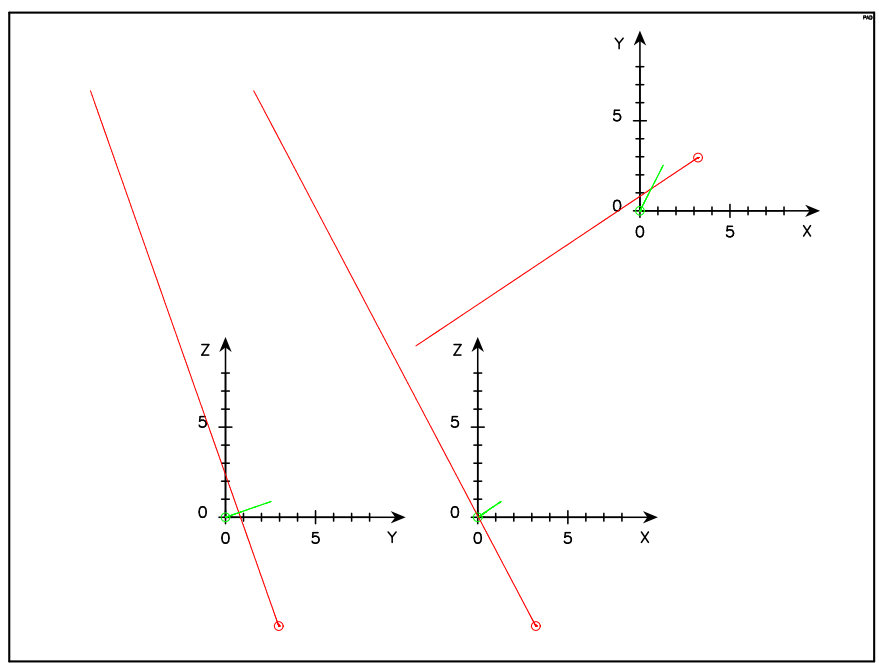

Fig. B.2. Geometry of the encounter of 'Oumuamua with the star GJ $427423 \mathrm{kyr}$ ago. $112 \mathrm{kyr}$ of motion of these bodies is shown here.

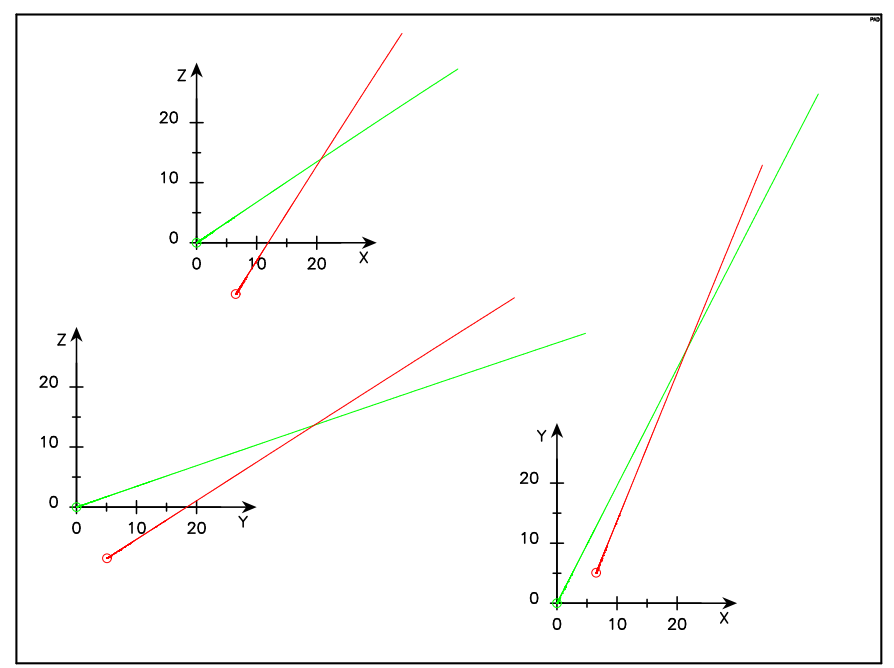

Fig. B.3. Geometry of the encounter of 'Oumuamua with the star $\delta$ Capricorni 1.5 Myr ago. Their past motion over 3.74 Myr is shown.

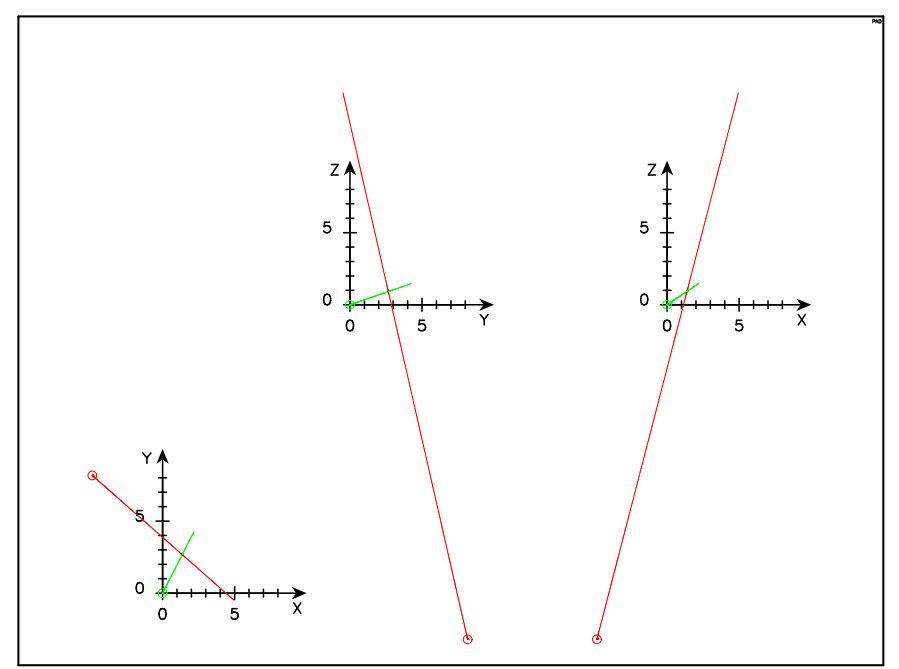

Fig. B.4. Geometry of the encounter of 'Oumuamua with the star HIP 3757118 kyr ago. $186 \mathrm{kyr}$ of motion of these bodies is shown here.

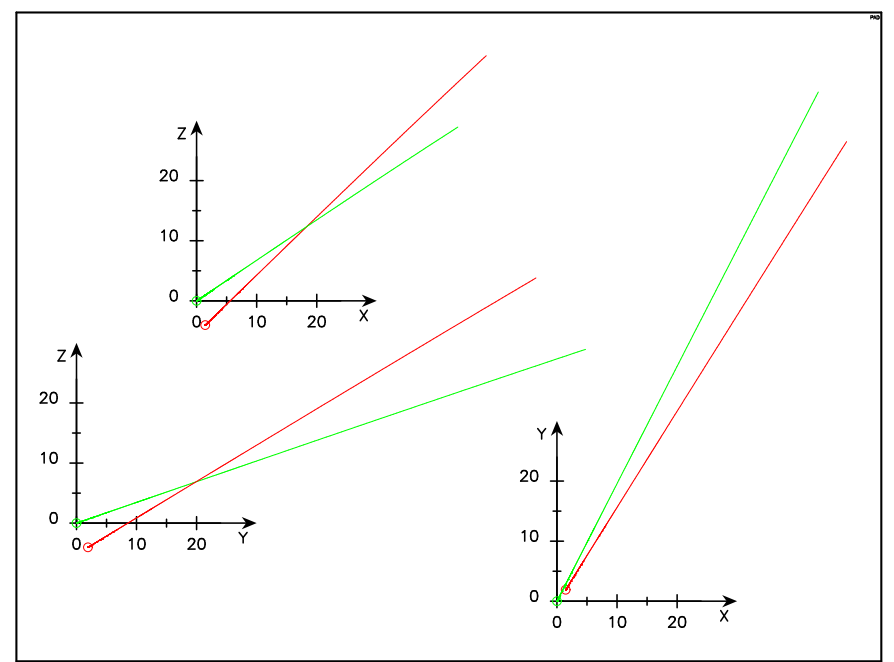

Fig. B.5. Geometry of the encounter of 'Oumuamua with the star HIP $1130200.8 \mathrm{Myr}$ ago. Past motion during 3.74 Myr is shown. 
A\&A 610, L11 (2018)

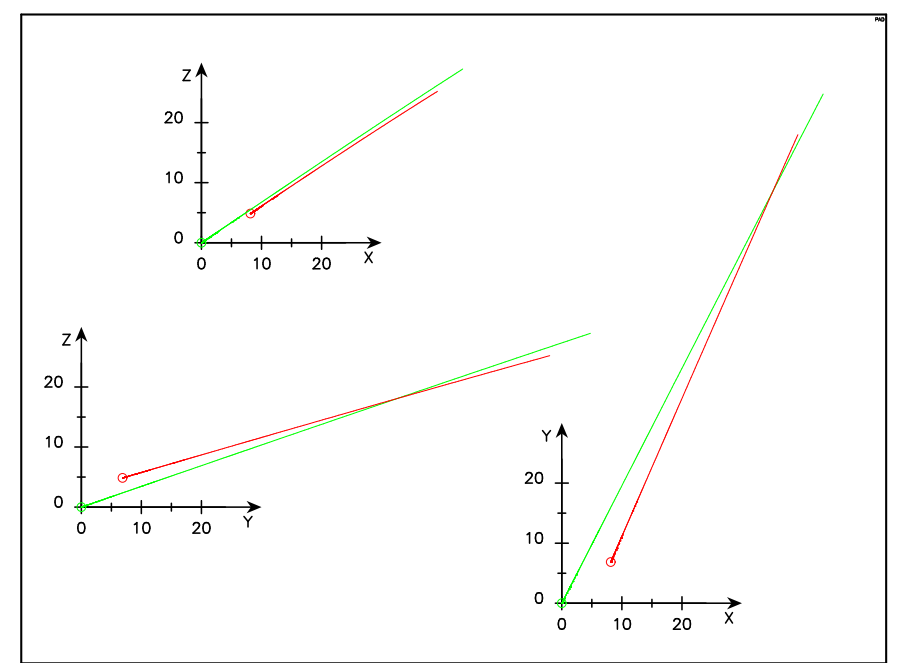

Fig. B.6. Geometry of the encounter of 'Oumuamua with the star UCAC4 535-065571 2.14 Myr ago. Past motion during 3.74 Myr is shown. 